\title{
A new family of complex rotation-covariant multiresolution bases in $2 \mathrm{D}$
}

\author{
Brigitte Forster, Thierry Blu and Michael Unser \\ Biomedical Imaging Group, Swiss Federal Institute of Technology Lausanne, Switzerland
}

\begin{abstract}
We present complex rotation-covariant multiresolution families aimed for image analysis. Since they are complexvalued functions, they provide the important phase information, which is missing in the discrete wavelet transform with real wavelets.

Our basis elements have nice properties in Hilbert space such as smoothness of fractional order $\alpha \in \mathbb{R}^{+}$. The corresponding filters allow a FFT-based implementation and thus provide a fast algorithm for the wavelet transform.
\end{abstract}

Keywords: Complex wavelets, Riesz basis, two-scale relation, multiresolution, scaling functions

\section{COMPLEX WAVELET APPROACHES}

The discrete wavelet transform (DWT) with real wavelets became an essential tool for signal and image analysis because of its multiresolution property and the existence of fast algorithms. Up to now only a few researchers have experimented with complex wavelet approaches, although these allow to introduce phase information. The phase provides a description of the local behavior of the image. So far, the approaches concentrate on filter design. ${ }^{1-3}$ The wavelet is calculated afterwards from the filter coefficients by iterative methods. This often results in wavelets with unfavorable properties, e.g. fractal-like functions. Other approaches consider redundant complex wavelet transforms ${ }^{4,5}$ and abandon DWT environment and multiresolution analyses.

We examine another approach to construct complex-valued multiresolution bases and consider a family of complex 2D rotation-covariant functions. Convolution with appropriate $2 \pi \mathbb{Z}^{2}$-periodic functions yields scaling functions in the Hilbert space $L^{2}\left(\mathbb{R}^{2}\right)$. They generate complex multiresolution analyses. Both, scaling functions and the corresponding wavelets have explicit analytic representations, and an arbitrary degree of smoothness. The respective discrete wavelet transform is implemented in Fourier space and yields a fast and stable algorithm.

\section{LOCALIZED ROTATION-COVARIANT FUNCTIONS}

The classical approach to a $2 \mathrm{D}$ wavelet basis decomposition for image analysis consists in using tensor products of a 1D scaling function $\varphi$ and a corresponding wavelet $\psi$. This analysis method tends to privilege the ordinates and image details in the vertical and horizontal directions.

It is now well established that the discrete wavelet transform is a powerful and nearly optimal tool for the analysis of piecewise smooth functions in one dimension. Unfortunately, for the reasons exposed before, the situation is not as favorable in higher dimensions. Classical tensor product wavelets have several disadvantages when applied to image analysis or higher dimensions. The most prominent one is directional selectivity. In addition, hitherto used real wavelets do not provide phase information. The phase provides a description of the local behavior of an image. Furthermore, the amplitude-phase representation imparts greater robustness to image processing algorithms.

Further author information: (Send correspondence to B.F.)

B.F.: E-mail: brigitte.forster@epfl.ch, Telephone: +41 (21) 6935172 ,

T.B.: E-mail: thierry.blu@epfl.ch, Telephone: +41 (21) 6935172 ,

M.U.: E-mail: michael.unser@epfl.ch, Telephone: +41 (21) 6935175 ,

Address: Biomedical Imaging Group, EPFL LIB, Bât. BM 4.127, Swiss Federal Institute of Technology Lausanne, CH-1015 Lausanne, Switzerland 
In contrast to the classical construction method, here we consider the function space

$$
V_{0}=\operatorname{span}_{k \in \mathbb{Z}^{2}}\{\rho(x-k)\} \cap L^{2}\left(\mathbb{R}^{2}\right),
$$

which is generated by a rotation-covariant function $\rho$ in the complex plane. Here rotation-covariance means $\rho\left(R_{\theta} x\right)=e^{i N \theta} \rho(x)$ for the rotation operator $R_{\theta}$ and $N \in \mathbb{N}$. We define $\rho$ in the sense of distributions by

$$
\widehat{\rho}\left(\omega_{1}, \omega_{2}\right):=\frac{1}{\left(\omega_{1}^{2}+\omega_{2}^{2}\right)^{\alpha}\left(\omega_{1}-i \omega_{2}\right)^{N}} \in \mathcal{D}_{L^{2}\left(\mathbb{R}^{2} \backslash\{(0,0)\}\right)}^{\prime}
$$

where $\left(\omega_{1}, \omega_{2}\right) \in \mathbb{R}^{2} \backslash\{(0,0)\}, \alpha \in \mathbb{R}^{+}, N \in \mathbb{N}$.

To localize the generating function $\rho$, we eliminate the singularity of $\hat{\rho}$ at the origin by multiplying the function by an appropriate bounded $(2 \pi, 2 \pi)$-periodic function $\nu$. For example, we found that the trigonometric polynomial

will do. Then

$$
\nu\left(\omega_{1}, \omega_{2}\right)=\left(4\left(\sin ^{2} \frac{\omega_{1}}{2}+\sin ^{2} \frac{\omega_{2}}{2}\right)\right)^{\alpha+\frac{N}{2}}
$$

$$
\widehat{\varphi}\left(\omega_{1}, \omega_{2}\right)=\frac{\nu\left(\omega_{1}, \omega_{2}\right)}{\left(\omega_{1}^{2}+\omega_{2}^{2}\right)^{\alpha} \cdot\left(\omega_{1}-i \omega_{2}\right)^{N}}
$$

is bounded in $\left(\omega_{1}, \omega_{2}\right)=(0,0)$. Moreover, the function is square-integrable for $\alpha+\frac{N}{2}>\frac{1}{4}$ and has fast decay $\left|\widehat{\varphi}\left(\omega_{1}, \omega_{2}\right)\right|=\mathcal{O}\left(\left\|\left(\omega_{1}, \omega_{2}\right)\right\|^{-2 \alpha-N}\right)$ for $\left\|\left(\omega_{1}, \omega_{2}\right)\right\| \rightarrow \infty$. This results in a function $\varphi\left(x_{1}, x_{2}\right) \in L^{2}\left(\mathbb{R}^{2}\right)$ with explicit time representation $\varphi(x)=\sum_{k \in \mathbb{Z}^{2}} \nu_{k} \rho(x+k)$ for almost all $x=\left(x_{1}, x_{2}\right) \in \mathbb{R}^{2}$. Here $\left(\nu_{k}\right)_{k \in \mathbb{Z}}$ denotes the sequence of Fourier coefficients of the $(2 \pi, 2 \pi)$-periodic multiplier $\nu\left(\omega_{1}, \omega_{2}\right)$ and $\rho$ is the inverse Fourier transform of the Hadamard partie finie $P f \widehat{\rho}$ :

$$
\rho\left(x_{1}, x_{2}\right)=\left(x_{1}^{2}+x_{2}^{2}\right)^{\alpha-1}\left(x_{1}-i x_{2}\right)^{N} \cdot C(\alpha, N) \text { for } \alpha \notin \mathbb{N},
$$

with constant $C_{\alpha, N}=\frac{1}{4} \pi^{2(\alpha+N-1)-1}(-2 i)^{N} \Gamma(-(\alpha+N)+1) \Gamma(\alpha+N-1) \Gamma(\alpha+N)^{-1} \Gamma(\alpha-1)^{-1}$. For $\alpha \in \mathbb{N}$ the term has to be modified by a factor $\ln \left(\sqrt{x_{1}^{2}+x_{2}^{2}}\right) c(\alpha, N), c(\alpha, N)$ constant. This can be deduced by passing from the nonsingular values to the limit $\alpha \in \mathbb{N}$. Moreover, in defining $r:=\sqrt{x_{1}^{2}+x_{2}^{2}}$ and $\theta:=\arctan \frac{x_{2}}{x_{1}}$ the function $\rho$ can be represented in polar coordinates

$$
\rho(r, \theta)=r^{2 \alpha+N-2} e^{-i N \theta} \cdot C^{\prime}(\alpha, N) \text { for } \alpha \notin \mathbb{N},
$$

which shows clearly the rotation-covariance. Here again, $C^{\prime}(\alpha, N)$ is a constant depending on $\alpha$ and $N$, and in the case $\alpha \in \mathbb{N}$ the formula has to be modified by a factor $\ln r$. From the decay properties of the Fourier transform in frequency we deduce that the function $\varphi(x)$ is an element of the Sobolev space $W_{2}^{m}\left(\mathbb{R}^{2}\right)$ for all $m<2 \alpha+N-1$.

\section{MULTIRESOLUTION BASES}

The function $\varphi$ satisfies a refinement relation for a family of dilation matrices and generates a multiresolution analysis. Consider the dilation matrices $A \in G L(2, \mathbb{R})$ of the form

$$
A=\left(\begin{array}{rr}
a & b \\
-b & a
\end{array}\right), \quad a, b \in \mathbb{Z},
$$

with eigenvalues of absolute value greater than one. This includes the quincunx case. The function $\varphi$ satisfies an $A$-scale relation for all those matrices. For $\omega=\left(\omega_{1}, \omega_{2}\right)^{T}$ the refinement filter has the following form:

$$
\begin{aligned}
H\left(e^{i \omega}\right) & =\frac{\widehat{\varphi}\left(A^{T} \omega\right)}{\widehat{\varphi}(\omega)}=\frac{\widehat{\rho}\left(A^{T} \omega\right) \nu\left(A^{T} \omega\right)}{\widehat{\rho}(\omega) \nu(\omega)} \\
& =\frac{\nu\left(A^{T} \omega\right)}{\nu(\omega)} \cdot \frac{1}{\left(a^{2}+b^{2}\right)^{\alpha}(a-i b)^{N}} .
\end{aligned}
$$

Theorem 3.1. Let $\alpha \in \mathbb{R}^{+}$and $N \in \mathbb{N}$ be fixed. Let the multiplier $\nu\left(\omega_{1}, \omega_{2}\right)$ be a bounded, $(2 \pi, 2 \pi)$-periodic function in $\mathbb{R}^{2}$ that fulfills the following properties: 
(i) $\left|\frac{\nu\left(\omega_{1}, \omega_{2}\right)}{\left(\omega_{1}^{2}+\omega_{2}^{2}\right)^{\alpha}\left(\omega_{1}-i \omega_{2}\right)^{N}}\right| \rightarrow c$ for $\left\|\left(\omega_{1}, \omega_{2}\right)\right\| \rightarrow 0$, and a positive constant $c$.

(ii) $\nu\left(\omega_{1}, \omega_{2}\right) \neq 0$ for all $\left(\omega_{1}, \omega_{2}\right) \in[-\pi, \pi]^{2} \backslash\{(0,0)\}$.

Then $\widehat{\varphi}=\nu \cdot \widehat{\rho}$ is the Fourier transform of a scaling function $\varphi$ which generates a multiresolution analysis $\left\{V_{j}\right\}_{j \in \mathbb{Z}}$ of $L^{2}\left(\mathbb{R}^{2}\right)$ with dilation matrix $A$ :

$$
V_{j}=\overline{\operatorname{span}\left\{|\operatorname{det} A|^{j / 2} \varphi\left(A^{j} \bullet-k\right), k \in \mathbb{Z}^{2}\right\}} .
$$

This can be proved by verifying that $\varphi$ satisfies the two-scale relation (cf. (1)), the Riesz basis condition, and by providing density arguments for the completeness of the multiresolution bases. In particular, we show that the second condition is met by bounding the autocorrelation filter $M(\omega):=\sum_{k \in \mathbb{Z}^{2}}|\widehat{\varphi}(\omega+2 \pi k)|^{2}$ :

$$
0<c \leq \sum_{k \in \mathbb{Z}^{2}}|\widehat{\varphi}(\omega+2 \pi k)|^{2} \leq C+B\left(\frac{2 \pi}{4 \alpha+2 N-2}+4 \zeta(4 \alpha+2 N)+\frac{1}{2^{4 \alpha+2 N}}\right)
$$

where $c$ is the minimum of $\left|\frac{\nu\left(\omega_{1}, \omega_{2}\right)}{\left(\omega_{1}^{2}+\omega_{2}^{2}\right)^{\alpha}\left(\omega_{1}-i \omega_{2}\right)^{N}}\right|, C$ the maximum of the same term, and $B$ the maximum of $|\nu|$ in $[-\pi, \pi]^{2}$ respectively. The function $\zeta$ denotes the Riemann zeta function.

\section{WAVELETS}

The bounded autocorrelation filter $M$ allows orthonormalization. We thus can construct explicitly all kinds of wavelets: orthonormal, semi-orthogonal or bi-orthogonal bases. The number of $\operatorname{cosets} q=|\operatorname{det} A|$ of $A\left(\mathbb{Z}^{2}\right)$ in $\mathbb{Z}^{2}$ indicates that $q-1$ wavelets are needed to span the orthogonal complement $W_{-1}=V_{0} \ominus V_{-1}$. A general method to construct the corresponding wavelets uses unitary polyphase matrices..$^{7,8}$

As an example we consider bi-orthogonal bases in the quincunx case $A=\left(\begin{array}{rr}1 & 1 \\ -1 & 1\end{array}\right)$ with $|\operatorname{det} A|=2$ and only one wavelet $\psi$ spanning the space $W_{-1}$. One possible choice is

$$
\widehat{\psi}\left(A^{T} \omega\right)=e^{-i \omega_{1}} \overline{H\left(\omega+(\pi, \pi)^{T}\right)} M\left(\omega+(\pi, \pi)^{T}\right) \widehat{\varphi}(\omega),
$$

such that

$$
W_{j}=\overline{\operatorname{span}\left\{\psi_{j, k}=2^{j / 2} \psi\left(A^{j} \bullet-k\right), k \in \mathbb{Z}^{2}\right\}} .
$$

Since $H\left(\omega+(\pi, \pi)^{T}\right)=\frac{\nu\left(\omega_{1}-\omega_{2}, \omega_{1}+\omega_{2}\right)}{\nu\left(\omega_{1}+\pi, \omega_{2}+\pi\right)}=\mathcal{O}\left(\left(\omega_{1}^{2}+\omega_{2}^{2}\right)^{\alpha+\frac{N}{2}}\right)$ for $\left\|\left(\omega_{1}, \omega_{2}\right)\right\| \rightarrow 0$, the Fourier transform of the wavelet $\widehat{\psi}$ has a rotation-covariant behavior in a neighborhood of $(0,0)^{T}$ :

$$
\widehat{\psi}(\omega)=\mathcal{O}\left(\left(\omega_{1}^{2}+\omega_{2}^{2}\right)^{\alpha}\left(\omega_{1}+i \omega_{2}\right)^{N} M\left((\pi, \pi)^{T}\right)\right) .
$$

This implies that the wavelet transform of a test function $f$,

$$
\begin{aligned}
& \langle f, \psi(\bullet-k)\rangle=\frac{1}{(2 \pi)^{2}}\langle\widehat{f}, \widehat{\psi(\bullet-k)}\rangle \\
= & \frac{1}{(2 \pi)^{2}} \int_{\mathbb{R}^{2}} \widehat{f}(\omega) e^{i\langle\omega, k\rangle} \overline{\widehat{\psi}(\omega)} d \omega \\
= & \frac{1}{(2 \pi)^{2}} \int_{\mathbb{R}^{2}} \widehat{f}(\omega)\left(\omega_{1}^{2}+\omega_{2}^{2}\right)^{\alpha}\left(\omega_{1}-i \omega_{2}\right)^{N} \overline{\widehat{\Phi}(\omega)} e^{i\langle\omega, k\rangle} d \omega \\
= & (-\Delta)^{\alpha}\left(-i \frac{\partial}{\partial x_{1}}-\frac{\partial}{\partial x_{2}}\right)^{N}\{f * \bar{\Phi}\}(k)
\end{aligned}
$$


behaves as a Laplacian of order $\alpha$ modified by a differential operator of Wirtinger type of order $N$. Here $\widehat{\Phi}(\omega)=\frac{\widehat{\psi}(\omega)}{\left(\omega_{1}^{2}+\omega_{2}^{2}\right)^{\alpha}\left(\omega_{1}+i \omega_{2}\right)^{N}}=e^{-\frac{i}{2}\left(\omega_{1}+\omega_{2}\right)}(1+\mathcal{O}(\|\omega\|)) M\left(A^{-T} \omega+(\pi, \pi)^{T}\right) \widehat{\varphi}\left(A^{-T} \omega\right)$ for $\|\omega\| \rightarrow 0$ is a lowpass kernel. The wavelet thus can be represented as

$$
\psi\left(x_{1}, x_{2}\right)=(-\Delta)^{\alpha}\left(-i \frac{\partial}{\partial x_{1}}-\frac{\partial}{\partial x_{2}}\right)^{N} \overline{\Phi\left(x_{1}, x_{2}\right)}
$$

i.e., the derivative of order $2 \alpha+N$ of the kernel $\bar{\Phi}$.

\section{IMPLEMENTATIONAL ASPECTS}

The coefficients of the refinement filter $H$ depend on the choice of the $(2 \pi, 2 \pi)$-periodic localizing function $\nu$. Moreover, for non-integer $\alpha$, the filter $H$ has in general an infinite impulse response and is non-causal. Therefore, a classical implementation by convolution in space domain would tend to be rather costly and would lead to accuracy problems because of the necessity to truncate the filters.

Since the filter $H$ is given explicitly in Fourier domain (1), it is more convenient to implement the corresponding wavelet transform in the Fourier domain using FFTs. We again consider the quincunx case, where we have to take into account just one wavelet filter. The discrete Fourier transform of the image data is filtered by multiplying with the refinement and the wavelet filter. Exploiting symmetries, we can downsample the data by a factor of 2 and use an inverse discrete Fourier transform on the reduced data. This yields a fast and stable algorithm.

\section{CONCLUSION}

We presented a new family of complex multiresolution bases in $L^{2}\left(\mathbb{R}^{2}\right)$. Our approach considered localizations of the rotation-covariant function of the form $\rho\left(x_{1}, x_{2}\right)=C(\alpha, N)\left(x_{1}^{2}+x_{2}^{2}\right)^{\alpha-1}\left(x_{1}+i x_{2}\right)^{N}($ times a logarithmic factor if $\alpha \in \mathbb{N})$. This yields nonseparable complex-valued multiresolution bases of $L^{2}\left(\mathbb{R}^{2}\right)$ for scaled rotation dilation matrices.

The parameter $\alpha \in \mathbb{R}^{+}$can be chosen arbitrarily; in particular, non-integer. This gives flexibility: We can control the smoothness and decay of the resulting wavelets. With the integer parameter $N$, we are able to influence rotation-covariance properties. Both degrees of freedom can be important for applications in image analysis.

Since our new family of complex rotation-covariant functions yields multiresolution bases, we can apply them in the DWT algorithm. We thus have a non-redundant complex wavelet transform and perfect reconstruction. Furthermore, there is not much change for the implementation in comparison to the real DWT: Our transform can still be implemented using a perfect reconstruction filterbank. The wavelet filters can also be specified to yield various types of decompositions; i.e., orthogonal, semi-orthogonal, or bi-orthogonal. Moreover, our FFT-based implementation provides a fast algorithm.

\section{ACKNOWLEDGMENTS}

The first author is supported by the grant BMBF-LPD 9901/8-64 from the German Academy of Natural Scientists Leopoldina by means of the German Federal Ministry of Education and Research.

\section{REFERENCES}

1. W. Lawton, "Applications of complex valued wavelet transforms to subband decomposition," IEEE Transactions on Signal Processing 41(12), pp. 3566-3568, 1993.

2. J.-M. Lina and M. Mayrand, "Complex Daubechies wavelets," Applied and Computational Harmonic Analysis 2, pp. 219-229, 1995.

3. X.-P. Zhang, M. D. Desai, and Y.-N. Peng, "Orthogonal complex filter banks and wavelets: Some properties and design," IEEE Transactions on Signal Processing 47, pp. 1039-1048, April 1999. 
4. N. Kingsbury, "Image processing with complex wavelets," Phil. Trans. R. Soc. Lond. A 357, pp. 2543-2560, 1999.

5. I. W. Selesnick, "Hilbert transform pairs of wavelet bases," IEEE Signal Processing Letters 8, pp. 170-173, June 2001.

6. L. Schwartz, Théorie des Distributions, Hermann, 1998. Reprint. Original of 1966.

7. P. Wojtaszczyk, A Mathematical Introduction to Wavelets, no. 37 in London Mathematical Society Student Texts, Cambridge University Press, 1997.

8. I. Daubechies, Ten lectures on wavelets, CBMS-NSF Regional Conference series in applied mathematics, Society for Industrial and Applied Mathematics, 1992. 